\title{
A Scalable and Fault Tolerant Health Risk Predictor using Bigdata Process Systems
}

\author{
Timmana Hari Krishna, C Rajabhushanam, D.Jayapriya, S.Deivasigamani
}

\begin{abstract}
: fault tolerant system to do real-time analytics for different health care applications. Users can get their health condition analysis report from the system by sending their health records in real-time. The health conditions occurrence can be considered as complex events and it may extended to different heterogeneous scenarios. Based on scalability and availability requirements, the system is developed using Kafka, Spark Streaming and Cassandra and implemented by using Scala. This system is capable for event stream processing and event batch processing. Users send the health data to Kafka through their producer clients in real-time. Spark streaming process the data from Kafka of different window sizes by analyzing the health conditions. In another scenario, user request stored into Cassandra database and is processed asynchronously by spark streaming. This system is tested with the use case of Heart attack hazard and stress prediction with different health datasets Keywords-Healthcare, Bigdata, Spark Streaming, Kafka, cassandra, Heart failure Prediction, Stress Index analysis

Keywords : Implementation, Casagranda database, Sparkstreaming
\end{abstract}

\section{INTRODUCTION}

In these days, real time health data collection is very common with various inexpensive health monitoring systems. This data is immersive and processed by using numerous signal processing and machine learning techniques[1]. This process is similar in feature identification, extraction and inferences across different applications Researchers are working on generalization of pre-processing and processing techniques prior to make inferences [3]. The collected health data can be processed in real time and offline to develop multiple inferences about the patient records [5].

In recent years, internet applications are extended in all the domains. In health care domain, applications are very limited due to processing and network demands on the

Revised Manuscript Received on July 22, 2019.

Timmana Hari Krishna, Department of Computer Science and Engineering, Bharath Institute of Higher Education and Research, Chennai. Email: harikrishna.timmana@gmail.com

C Rajabhushanam, Department of Computer Science and Engineering, Bharath Institute of Higher Education and Research, Chennai. Email: rajabhushanamc.cse@bharathuniv.ac.in

D,Jayapriya, Department of Computer Science and Engineering, Bharath Institute of Higher Education and Research, Chennai. Email: priyajp8@gmail.com

S.Deivasigamani, Department of Computer Science and Engineering, Bharath Institute of Higher Education and Research, Chennai. Email: dheiva94@gmail.com supportive infrastructure. It requires a real world application to analyze high resolution sensor data in real-time and offline from heterogeneous sources simultaneously. The system should handle requests from huge number of users concurrently with high availability. This can be achieved by using huge distributed processing frames works like Kafka, Spark Streaming and Cassandra

In recent years, internet applications are extended in all the domains. In health care domain, applications are very limited due to processing and network demands on the supportive infrastructure. It requires a real world application to analyze high resolution sensor data in real-time and offline from heterogeneous sources simultaneously. The system should handle requests from huge number of users concurrently with high availability. This can be achieved by using huge distributed processing frames works like Kafka, Spark Streaming and Cassandra

It requires a real world application to analyze high resolution sensor data in real-time and offline from heterogeneous sources simultaneously. The system should handle requests from huge number of users concurrently with high availability. This can be achieved by using huge distributed processing frames works like Kafka, Spark Streaming and Cassandra

Recently, there has been a great consideration to improve the efficiency of the system through system implementation and optimization of algorithms [7, 12, 13]. However, these methods only recommend solutions for certain problems. They include design choices that are hard to generalize owe to problem specific assumptions. To discourse this issue of application dependent implementations there is a need for processing platforms that are effective enough to function under real-world hardware and software limitations. Also, at the same time to be broad enough to support different problems and applications. This work proposes an architecture that solves this problem using intellectual distribution of the computational weight using a publication/subscription scheme.

In this concern, the objective is to design the system which can scale to handle enormous number of users and can exploit as a platform for processing real-time health analytics and inferences. We have developed the system using apache Kafka, apache Spark 
and Cassandra. All these three systems can run on same cluster and can be scaled simply. These are fault tolerant with high availability. To assess the system, we used the real time health data repositories and executed the real-time heart risk and stress prediction use cases. It uses the publish-subscribe systems and clustered processing framework to develop the complex event processing on top of health data.

This paper is organized as follows: In section II, we present the system architecture and its components. In section III, we discuss about the two health care use cases in details. In section IV, we refer the related work done for doing experiments. In section $\mathrm{V}$, we discuss about the experiment with results. Finally, we consider the future effort with conclusion.

\section{SYSTEM DESIGN}

This system follows lambda architecture which is designed to handle massive quantity of data by delightful features of both batch processing and stream processing. Lambda architecture consists of following three layers a) Batch layer which performs write once and mass read many times. b) Service layer which performs random read with no random write, batch calculation and batch write. C) Speed Layer which performs random read and write with incremental calculation

This System is designed using Kafka, Spark Streaming and Cassandra and implemented using Scala. Kafka Topic is used to get the user data and send to the spark stream as streaming data. Spark Streaming API process and analyze the streaming data to predict the stress index and heart failure risk over the windows of data streams. Spark Streaming API also process the user requests saved in Cassandra

\section{A. Apache Spark}

Apache spark [4] is a big data in-memory processing framework that runs on multiple clusters. It provides good reliability and fault tolerance along with in-memory processing features. The different regulated transformations and queries are supported on the datasets, which are essentially parallel and fault tolerant. Spark contains following modules a) core library supports basic Input and output operations along with core processing b) SQL and data frame library supports SQL related operations. C) Streaming library which supports scalable streaming applications d) Mlib library which provides Machine learning algorithm support. e) GraphX library provides supports for graph processing. Streaming supports data insertion from multiple sources like Kafka, Flume, Hadoop Distributed File System, Amazon S3, Kinesis, Twitter and etc.. It also affords an abstraction of DStreams which encloses batches of data produced from the real-time streaming source. The DStream is represented as a sequence of resilient distributed datasets to perform the basic spark operations.

\section{B. Apache Kafka}

Apache Kafka [6] is a streaming platform which permits users to publish data and also subscribe to different streams of records. Kafka handles the streams in a fault tolerant manner. It is used to construct reliable real-time streaming data pipelines. Further, it runs as a cluster and implements the concept of topics or feed name which records are published to. In existing system abstraction, each user is publishing its data to a particular topic chosen for the stream. Each topic preserves all the records internally for the configurable retention period. This feature is used for health conditions that can be analyzed with past data. The anatomy of Kafka Topic is as shown in Fig .1

\section{Anatomy of a Topic}

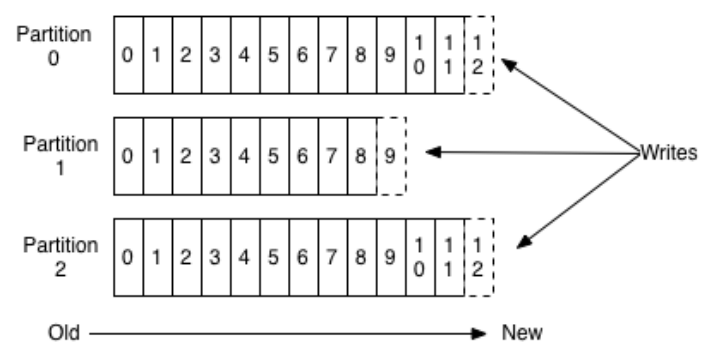

\section{Cassandra}

Cassandra[9] is a highly scalable distributed and decentralized database from Apache thatprovides high availability with no single point of failure. It is designed to manage very large amounts of structured data across many commodity servers. It has following features a)Accommodates more customers and data by adding more hardware. b) Highly available through no single point of failure c)Increases throughput by increasing the number of nodes in the cluster. d) It accommodates structured, semi structured and unstructured data formats. e) Provides the flexibility to distribute data by replicating data across multiple data centers. f)Supports Atomicity, Consistency, Isolation, and Durability. g) Performs fast writes and can store hundreds of terabytes of data, without losing the read efficiency[10-15]

\section{PROPOSED ARCHITECTURE}

The proposed system architecture is as shown in the fig. 2 . Multiple users act as data producers and consumers in the Kafka model. Users publishes their health data for further processing. In this system the assumption is that users are aware of the different topics available for different types of sensory data. For example ECG data might be published to a topic dedicated to it, and BP data to a different topic and so-on. Kafka takes care of the scalability of the system as the number of users may grow or shrink. It also serves as a data retention, storage and forwarding interface.[16-20] 


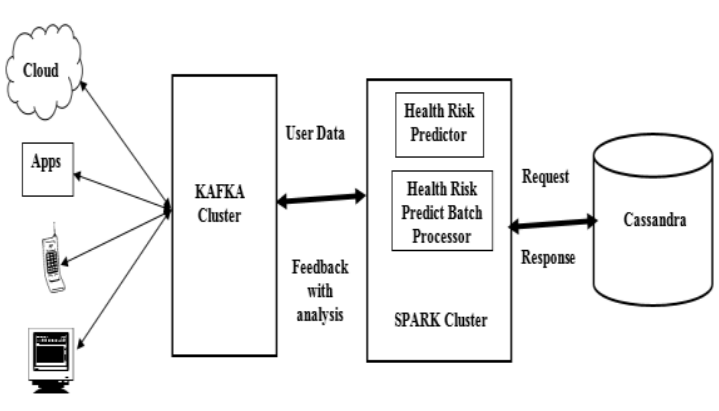

Fig2. Proposed System Architecture

Different types of complex events defining the health conditions are running on the Spark cluster. For example in figure 2 we have shown two such events of heart failure risk and stress index detection. These events are running as separate jobs using Spark streaming on the Spark cluster. Thus this system is extensible where multiple such jobs can be created or aborted depending on the de-sired analytics to be processed. Since, these jobs are running on the spark cluster they are scalable and are fault tolerant[21-25]. The complex events considered here are explained in more detail in the use cases section. Each of the jobs are internally subscribed to the relevant health data which is done by means of Spark streaming. DStream are created from the multiple Kafka topics and are processed in each of these. The real-time generated output can be saved to the database and can also be communicated back to the user via Kafka. For sending results back to users, the job publishes back to the Kafka. Different users are also subscribed to the Kafka to listen to the relevant topics for the analytics results.[25-30]

In other scenario, Users save the requests in Cassandra in JSON format. Health Predict batch processor job reads the user requests from HEALTH_PREDICTION _REQUESTS table and sent them for processing. Once the requests are processed and analyzed results will be saved in HEALTH_PREDICTION_RESULTS table in JSON format.

\section{A. Pipeline of Data}

The basic data pipeline for the heart risk failure is shown in figure 2. The processes used in the prediction for each use case are examined in more detail in the next section. The user publishes the ECG and BP data to the Kafka as JSON. A spark job acts Heart Risk predictor and uses spark streaming to continuously attend the Kafka topic for the user input data. There are two subscribed topics exists in the system. One is for BP and the other is for ECG. The Heart Risk Predictor publishes the results back to Kafka topic after completion of Processing and analysis. The sample data format of the ECG and BP is shown below and they are customizable.[31-34]

\section{EXAMPLE USE CASES}

In this section, we present two different use cases of the proposed system namely heart failure risk prediction and stress measurement from vital signals. We have used recorded Electrocardiograph (ECG) and Blood Pressure (BP) signals from the Physionet online dataset as a data source to simulate real-world complex event processing for each use case [8] Particularly, we have created two special Kafka producers corresponding to each signal (i.e., ECG and BP) to publish timestamp and signal amplitude as key value pairs with constant signal sampling rate of $500 \mathrm{~Hz}$.

\section{A. Heart Failure Risk Prediction}

Heart failure (HF) or congestive heart failure (CHF) happens when the heart is not efficient of pumping enough blood in the cardiovascular system that is vital for other body organs to work. CHF is one of the most collective hospitalization reasons among people aged 65 years and older [7]. Recently, there has been an excessive attention to early detection and treatment of CHF using vital signals [2].However, minute work has been done for real-time and online processing of these data sets. In this work, we present real-time $\mathrm{CHF}$ risk prediction as a potential use case of the proposed system.

On the data consumer side, we receive data from the ECG and BP producers in time windows of about 5 seconds. Prior to any analysis, signals of each window are preprocessed by statistically normalizing each window and applying appropriate Butterworth IIR filters. Then, informative features were extracted from each window. Specifically, we have measured the minima and maxima values of each BP signal window and averaged them together to calculate the diastolic blood pressure (DBP) and systolic blood pressure (SBP) values. Extracting features from the ECG signal is slightly more involved.[35] First, we normalize the ECG signal such that the minimum value is equal to zero and maximum value is equal to one. Then, the first derivative (i.e., difference) of the ECG signal is calculated and its zero crossing points are stored as potential local extreme points. Afterwards, ECG R-peaks inside the window are detected as the local maximums with the normalized amplitudes of more than 0.90 units. Finally, based on the comparative occurrence position of other ECG key-points with respect to the ECG R-peak, we have noticed P, Q, S, T time instances corresponding to each R-peak inside the window. We used the real-world vital signals which are significantly corrupted or noisy signal parts that makes feature extraction extremely hard. To handle this, we assigned negative numbers to the invalid feature values to indicate the issue rather than using the common exception handing methods. Processing blocks validate the extracted features by using these features before any further analysis.

- More than 80 bpm Heat-rate

- More than $100 \mathrm{~ms}$ QRS interval

- More than $120 \mathrm{~ms}$ QRS interval duration

- More than $410 \mathrm{~ms}$ QT interval

-ST depression pattern existence

-ST elevation pattern existence

-Inverted T-wave pattern existence

Here, different time interval values are easily measured using the time instances of the occurrence of each ECG key-point inside the window as explained. Different signal patterns like ST depression and elevation and inverted $\mathrm{T}$-wave patterns were detected by comparing the 
amplitude of each ECG key-point with respect to each other and also with the expected values for normal healthy ECG waveform. The exact analysis of each high-level feature and its contribution to $\mathrm{CHF}$ is discussed in the literature.[36]

At last, in order to predict the CHF risk values, we used a simple naive Bayes prediction algorithm to make the risk predictions. The model parameters were described based on the popularity of each high-level feature in CHF patients in the previous studies such asand [16]. Finally, we have standardized the predicted raw values using the maximum and minimum possible risk values such that the predicted values are representing the CHF risk percentages.

\section{B. Measuring Stress Index}

Stress is complex, as it is a subjective phenomenon and thus hard to define a precise measure. Stress is usually synonymous with distress, resulting from emotional, physical, and mental activities. It is the body's method of responding to activities a person under-takes, and hence the stress response of every individual is different. This queries for approaches capable of personalizing stress index to an individual person.

Stress is complex, as it is a subjective phenomenon and thus hard to define a precise measure. Stress is usually synonymous with distress, resulting from emotional, physical, and mental activities. It is the body's method of responding to activities a person under-takes, and hence the stress response of every individual is different. This queries for approaches capable of personalizing stress index to an individual person.

In the health literature there are several types of stress, acute and chronic stress. Acute (short term) stress effects from demands of recent activities and near future. On the other hand, chronic (long term) stress effects from pressure over long periods of times, which eventually lead to break down and unusual behavior of a person. The stress type and physiological cause of the person undergoing stress presents a challenge for processing. We predict short-term stress by using this use case.[38]

In the introduction, there has been challenges on quantifying short term stress using heart rate variability (HRV), and frequency do-main features of heart signals such as power in low frequency (LF) and power in high frequency (HF) $[11,10,15]$. It is renowned that as the body undergoes stress the autonomic nervous system (ANS) has a direct role in the physical response of the body. The ANS is classified as parasympathetic nervous system (PNS), and sympathetic nervous system (SNS). When the body undergoes stress due to physical activities, or mental load the SNS dominates the ANS. This results in lower HRV, and lower LF-HF ratio of the root mean square difference (RMMSD) of successive intervals extracted from the peaks of heart rate signals. In healthy conditions the PNS dominates the ANS and the respective values of $\mathrm{HRV}$ increases, and LF-LF ratio decreases. ECG signals are filtered and preprocessed as explained in the previous use case.[39]

The features to be extracted from each window are the peaks and HF, LF components. The R peaks are acquired using the peak detection algorithm as described previously.
The indices of the peaks are then used to compute the HRV using RMSSD as a measure given by.

Where $\mathrm{I}[\mathrm{x}]$ denotes the peak index. It is advised to use 1 minute windows for determining HRV values.

The RR series are got from consecutive windows are used to obtain the LF and HF components. The PSD of the RR series is attained using Fast Fourier Transform (FFT) method. Very low frequency (VLF, $0-0.4 \mathrm{~Hz}$ ), low frequency (LF, $0.4 \mathrm{~Hz}$ $.15 \mathrm{~Hz})$ and high frequency $(\mathrm{HF}, 0.15-0.4)$ are the commonly used bands. The LF component is computed by integrating the frequency amplitudes between the ranges of $0.04 \mathrm{~Hz}-$ $0.15 \mathrm{~Hz}$. Similarly, the HF component is computed in the range of $0.15 \mathrm{~Hz}-0.4 \mathrm{~Hz}$ [9]. The ratio of LF and HF specifies the short-term stress index. A high value is associated with high SNS activity, and a low value is associated with PNS dominant ANS.

To predict the stress, a base-line measure of the person can be obtained when he/she is sleeping. When the person is sleeping short term stressors are absent and hence a baseline can be taken for the day. The baseline is then used to compare HRV values obtained during the day. For distinguishing between physical stress, mental stress, and emotional stress it is essential to take the context into account. The context can be taken by using other sensors such as accelerometer, gyroscope, and GPS to determine the activities the person is undertaking. Given the context it is possible to differentiate between good stressors and bad stressors.

$$
R M S S D=\sqrt{\frac{\sum_{1}^{X} I[x]-I[x-1]}{X-1}}
$$

\section{IV.SPARK RDD OPERATIONS}

In this section, we will discuss how to define the use cases in the spark map reduce framework and the other transformations and operations supported by it. The objectives behind these definitions is that, these operations are highly parallel and fault tolerable. Also in future these operations may be individually relocated to different places for the reason of faster processing, reliability and security.[40]

Kafka producer sends the data in the JSON array format using the windows of sizes required for the heart risk measurement and stress index measurement. We formed several RDDs from the data received using the spark transformations. Logically each RDD has different stages in the signal processing and are equivalent to the peaks of the ECG and BP signals, along with different operations (i.e. transformations and actions) on it.

\section{EXPERIMENTS AND RESULTS}

The proposed system was developed in Scala. We simulated the multiple users by sending real-time data as JSON via different applications to the Kafka cluster. The implementation of both use cases is using Spark streaming in Scala, and each is being run as a separate job on the local Spark 
cluster. The jobs do the real-time health analytics and produce the heart risk and stress index as output. Figure 3 shows three different ECG signal windows equivalent to different individuals. For each signal, the P, Q, R, S, and T

ECG key-points are specified [18] with red circle markers inside each plot. Figure $4 \mathrm{a}$ is an ECG signal with the waveform related to a normal healthy person, and the predicted CHF risk by the proposed is about $1.2 \%$. Figure $4 \mathrm{~b}$ is associated to an ECG waveform with slightly abnormal $\mathrm{S}$ point, and the predicted risk in this case is about $12.5 \%$. Finally, $4 \mathrm{c}$ is a waveform with ST depression pattern and with the predicted risk of about $20.6 \%$. As it is evident from this example, the proposed system is able to predict realistic CHF risk values based on presented data in real-time.[30]

To calculate the stress index, the heart rate and HRV for each window is used. In this application the stress index corresponds to a change in HRV in consecutive windows. The consecutive difference of RR intervals is stored in a circular buffer before more windows are obtained. Once the circular buffer is filled, at least 1-minute duration worth of $\mathrm{RR}$ interval differences is stored and is used to calculate the HRV for the corresponding window. If the HRV drips below the baseline, SNS is taking over the ANS and hence the stress index is improved. If the HRV increases PNS is more dominant and the stress index decreases. In this application the baseline is set to be the HRV obtained from the previous window.[41]

TABLE I. SAMPLES OF STRESS MEASUREMENT USE CASE

\begin{tabular}{|l|l|l|l|}
\hline Window \# & HRV & HR & Stress Index \\
\hline 1 & 107.53 & 138.5 & 0.1 \\
\hline 2 & 105.00 & 143.3 & 0.2 \\
\hline 3 & 104.59 & 147.3 & 0.3 \\
\hline 4 & 98.86 & 148.73 & 0.4 \\
\hline 5 & 97.02 & 148.3 & 0.5 \\
\hline 6 & 98.47 & 143.3 & 0.4 \\
\hline
\end{tabular}

As shown in Table 1, the stress index starts at 0.1 , and increases to 0.5 before dropping back down. This is owed to decrease in the HRV value, whereas HR is relatively constant.

As the major effort of this work is to present an overall architecture for the CEP implementation on mobile systems and use cases are used as models that run on this system, detailed analysis and evaluation of the proposed use cases is out of the scope.

\section{FUTURE WORK}

In the application, we have executed the two health use cases. It proves that a common platform is capable of processing other applications of health inference and behavioral patterns. For instance, real-time prediction of smoking can be implemented using the proposed system architecture. We would also like to test this system on real scenarios, as in proposed implementation we simulated the user data using real datasets by running separate client applications. More work can also be done to state the complex events in generalized way for the health applications, so that the different signal processing potentials can be reused. A simple and expressive grammar can be suggested for the same which achieves the job of events semantic definitions, the required inputs, outputs and logic. However, such a goal involves effort to appreciate the nature of different health analytics to develop a generalization.

\section{VII.CONCLUSION}

In this paper, we developed a framework to perform health analytics in real-time. By developing the framework using Kafka,Spark and Cassandra which handle large amounts of health data reliably and efficiently. Components of system are highly scalable and available. We implemented a model system for running two use cases in real-time and offline. This can be extended to new use cases by developing separate Spark job .This design can be improved by enhancing the data security and access control features so as to deploy with real users or to conduct research.

\section{REFERENCES}

[1] Kumaravel A., Rangarajan K.,Algorithm for automaton specification for exploring dynamic labyrinths,Indian Journal of Science and Technology,V-6,I-SUPPL5,PP-4554-4559,Y-2013

[2] P. Kavitha, S. Prabakaran "A Novel Hybrid Segmentation Method with Particle Swarm Optimization and Fuzzy C-Mean Based On Partitioning the Image for Detecting Lung Cancer" International Journal of Engineering and Advanced Technology (IJEAT) ISSN: 2249-8958, Volume-8 Issue-5, June 2019

[3] Kumaravel A., Meetei O.N.,An application of non-uniform cellular automata for efficient cryptography,2013 IEEE Conference on Information and Communication Technologies, ICT 2013,V-,I-,PP-1200-1205,Y-2013

[4] Kumarave A., Rangarajan K.,Routing alogrithm over semi-regular tessellations,2013 IEEE Conference on Information and Communication Technologies, ICT 2013,V-,I-,PP-1180-1184,Y-2013

[5] P. Kavitha, S. Prabakaran "Designing a Feature Vector for Statistical Texture Analysis of Brain Tumor" International Journal of Engineering and Advanced Technology (IJEAT) ISSN: 2249-8958, Volume-8 Issue-5, June 2019

[6] Dutta P., Kumaravel A.,A novel approach to trust based identification of leaders in social networks, Indian Journal of Science and Technology,V-9,I-10,PP--,Y-2016

[7] Kumaravel A., Dutta P.,Application of Pca for context selection for collaborative filtering,Middle - East Journal of Scientific Research,V-20,I-1,PP-88-93,Y-2014

[8] Kumaravel A., Rangarajan K.,Constructing an automaton for exploring dynamic labyrinths,2012 International Conference on Radar, Communication and Computing, ICRCC 2012,V-,I-,PP-161-165,Y-2012

[9] P. Kavitha, S. Prabakaran "Adaptive Bilateral Filter for Multi-Resolution in Brain Tumor Recognition” International Journal of Innovative Technology and Exploring Engineering (IJTEE) ISSN: 2278-3075, Volume-8 Issue-8 June, 2019

[10] Kumaravel A.,Comparison of two multi-classification approaches for detecting network attacks, World Applied Sciences Journal,V-27,I-11,PP-1461-1465,Y-2013

[11] Tariq J., Kumaravel A.,Construction of cellular automata over hexagonal and triangular tessellations for path planning of multi-robots,2016 IEEE International Conference on Computational Intelligence and Computing Research, ICCIC 2016,V-,I-,PP--,Y-2017

[12] Sudha M., Kumaravel A.,Analysis and measurement of wave guides using poisson method,Indonesian Journal of Electrical Engineering and Computer Science,V-8,I-2,PP-546-548,Y-2017

[13] Ayyappan G., Nalini C., Kumaravel A., Various approaches of knowledge 
transfer in academic social network,International Journal of Engineering and Technology,V-,I-,PP-2791-2794,Y-2017

[14] Kaliyamurthie, K.P., Sivaraman, K., Ramesh, S. Imposing patient data privacy in wireless medical sensor networks through homomorphic cryptosystems 2016, Journal of Chemical and Pharmaceutical Sciences92.

[15] Kaliyamurthie, K.P., Balasubramanian, P.C. An approach to multi secure to historical malformed documents using integer ripple transfiguration 2016 Journal of Chemical and Pharmaceutical Sciences92.

[16]A.Sangeetha,C.Nalini,"Semantic Ranking based on keywords extractions in the web", International Journal of Engineering \& Technology, 7 (2.6) (2018) 290-292

[17] S.V.GayathiriDevi,C.Nalini,N.Kumar,"An efficient software verification using multi-layered software verification tool "International Journal of Engineering \& Technology, 7(2.21)2018 454-457

[18]C.Nalini,ShwtambariKharabe,"A Comparative Study On Different Techniques Used For Finger - Vein Authentication", International Journal Of Pure And Applied Mathematics, Volume 116 No. 82017 , 327-333, Issn: 1314-3395

[19]M.S. Vivekanandan and Dr. C. Rajabhushanam, "Enabling Privacy Protection and Content Assurance in Geo-Social Networks", International Journal of Innovative Research in Management, Engineering and Technology, Vol 3, Issue 4, pp. 49-55, April 2018.

[20]Dr. C. Rajabhushanam, V. Karthik, and G. Vivek, "Elasticity in Cloud Computing", International Journal of Innovative Research in Management, Engineering and Technology, Vol 3, Issue 4, pp. 104-111, April 2018.

[21]K. Rangaswamy and Dr. C. Rajabhushanamc, "CCN-Based Congestion Control Mechanism In Dynamic Networks", International Journal of Innovative Research in Management, Engineering and Technology, Vol 3, Issue 4, pp. 117-119, April 2018.

[22] Kavitha, R., Nedunchelian, R., "Domain-specific Search engine optimization using healthcare ontology and a neural network backpropagation approach", 2017, Research Journal of Biotechnology, Special Issue 2:157-166

[23] Kavitha, G., Kavitha, R., "An analysis to improve throughput of high-power hubs in mobile ad hoc network" , 2016, Journal of Chemical and Pharmaceutical Sciences, Vol-9, Issue-2: 361-363

[24] Kavitha, G., Kavitha, R., "Dipping interference to supplement throughput in MANET" , 2016, Journal of Chemical and Pharmaceutical Sciences, Vol-9, Issue-2: 357-360

[25] Michael, G., Chandrasekar, A.,'Leader election based malicious detection and response system in MANET using mechanism design approach", Journal of Chemical and Pharmaceutical Sciences(JCPS) Volume 9 Issue 2, April - June 2016.

[26]Michael, G., Chandrasekar, A.,"Modeling of detection of camouflaging worm using epidemic dynamic model and power spectral density", Journal of Chemical and Pharmaceutical Sciences(JCPS) Volume 9 Issue 2, April - June 2016.

[27] Pothumani, S., Sriram, M., Sridhar, J., Arul Selvan, G., Secure mobile agents communication on intranet,Journal of Chemical and Pharmaceutical Sciences, volume 9, Issue 3, Pg No S32-S35, 2016

[28] Pothumani, S., Sriram, M., Sridhar, Various schemes for database encryption-a survey, Journal of Chemical and Pharmaceutical Sciences, volume 9, Issue 3, Pg NoS103-S106, 2016

[29]Pothumani, S., Sriram, M., Sridhar, A novel economic framework for cloud and grid computing, Journal of Chemical and Pharmaceutical Sciences, volume 9, Issue 3, Pg No S29-S31, 2016

[30] Priya, N., Sridhar, J., Sriram, M. "Ecommerce Transaction Security Challenges and Prevention Methods- New Approach” 2016 ,Journal of Chemical and Pharmaceutical Sciences, JCPS Volume 9 Issue 3.page no:S66-S68

[31] Priya, N.,Sridhar,J.,Sriram, M."Vehicular cloud computing security issues and solutions" Journal of Chemical and Pharmaceutical Sciences(JCPS) Volume 9 Issue 2, April - June 2016

[32] Priya, N., Sridhar, J., Sriram, M. "Mobile large data storage security in cloud computing environment-a new approach" JCPS Volume 9 Issue 2. April - June 2016

[33] Anuradha.C, Khanna.V, "Improving network performance and security in WSN using decentralized hypothesis testing "Journal of Chemical and Pharmaceutical Sciences(JCPS) Volume 9 Issue 2 , April - June 2016 .
[34] Anuradha.C, Khanna.V, "A novel gsm based control for e-devices" Journal of Chemical and Pharmaceutical Sciences(JCPS) Volume 9 Issue 2, April - June 2016 .

[35] Anuradha.C, Khanna.V, "Secured privacy preserving sharing and data integration in mobile web environments " Journal of Chemical and Pharmaceutical Sciences(JCPS) Volume 9 Issue 2, April - June 2016.

[36] Sundarraj, B., Kaliyamurthie, K.P. Social network analysis for decisive the ultimate classification from the ensemble to boost accuracy rates 2016 International Journal of Pharmacy and Technology

[37] Sundarraj, B., Kaliyamurthie, K.P. A content-based spam filtering approach victimisation artificial neural networks 2016 International Journal of Pharmacy and Technology83.

[38] Sundarraj, B., Kaliyamurthie, K.P. Remote sensing imaging for satellite image segmentation 2016 International Journal of Pharmacy and Technology8 3.

[39] Sivaraman, K., Senthil, M. Intuitive driver proxy control using artificial intelligence 2016 International Journal of Pharmacy and Technology84.

[40] Sivaraman, K., Kaliyamurthie, K.P. Cloud computing in mobile technology 2016 Journal of Chemical and Pharmaceutical Sciences92.

[41] Sivaraman, K., Khanna, V. Implementation of an extension for browser to detect vulnerable elements on web pages and avoid click jacking 2016 Journal of Chemical and Pharmaceutical Sciences92.

\section{AUTHORS PROFILE}

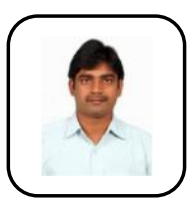

Timmana Hari Krishna Research Scholar, Department of Computer Science \& Engineering, Bharath Institute of Higher Education and Research, Chennai, India

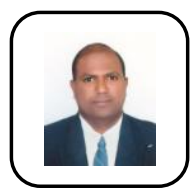

C Rajabhushanam Assistant Professor, Department of Computer Science \& Engineering, Bharath Institute of Higher Education and Research, Chennai, India

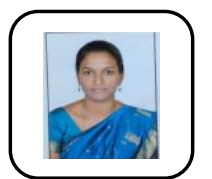

D.Jayapriya Assistant Professor, Department of Computer Science \& Engineering, Bharath Institute of Higher Education and Research, Chennai, India

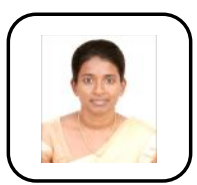

S.Deivasigamani Assistant Professor, Department of Computer Science \& Engineering, Bharath Institute of Higher Education and Research, Chennai, India. 